\title{
Aprendizajes clave y su impacto en el ámbito socioemocional del estudiante de nivel secundaria
}

\section{Key learning and its impact on the socioemotional area of the secondary level student}

\author{
VILLARREAL-SOTO, Blanca Margarita†*, RAMOS-JAUBERT Roció Isabel, CEPEDA-
} GONZALEZ, María Cristina y ESPERICUETA-MEDINA, Marta Nieves

Universidad Autónoma de Coahuila, Facultad. de Ciencia Educación y Humanidades

ID $1^{\text {er }}$ Autor: Blanca Margarita, Villarreal-Soto / ORC ID: 0000-0001-7435-415X, Reserche ID Thomsom: T-1500-2018, arXiv Author ID, Espericueta2018, CVU CONACYT ID: 372705

ID $1^{\mathrm{er}}$ Coautor: Roció Isabel, Ramos-Jaubert / ORC ID: 0000-0001-9314-8001, CVU CONACYT ID: 947979

ID $2^{\text {do }}$ Coautor: María Cristina, Cepeda-Gonzalez / ORC ID: 0000-0003-3289-5390, Researcher ID Thomson: T-16522018

ID $3^{\text {er }}$ Coautor: Marta Nieves, Espericueta-Medina / ORC ID: 0000-0003-0676-2412, CVU CONACYT ID: 567204

DOI: $10.35429 / \mathrm{JCP} .2019 .10 .3 .1 .8$

Recibido 13 de Agosto, 2019; Aceptado 30 de Diciembre, 2019

\section{Resumen}

El avance progresivo de las sociedades como resultado de la globalización, establece un escenario de competencia en el que el individuo debe buscar su mejora continua y el cumplimiento de estándares de profesionalización. Por lo que el objetivo de esta investigación es el conocer las repercusiones que tiene en la calidad educativa la aplicación del nuevo modelo educativo en las instituciones de educación secundaria. La muestra consto de 50 docentes de educación secundaria, con edades de entre los 23 y 45 años. Se aplicó un instrumento con 4 variables signalíticas y 36 variables del fenómeno de estudio, consideradas con una escala de razón decimal; las cuales se procesaron en los programas estadísticos SPSS y Statistica. Los principales resultados muestran que la evaluación del desempeño docente, es significativa para destacar la aplicación de procedimientos didácticos que aseguren el aprendizaje del estudiante. Además, a medida en que se propicie el desarrollo humano y la educación socioemocional se obtendrán resultados satisfactorios en la aplicación del nuevo modelo educativo. Por lo que se propone la realización de foros de mejora continua donde participen alumnos, docentes, directivos, padres de familia y agentes sociales que deriven en la creación de políticas públicas.

Calidad Educativa, Modelo Educativo, Docencia

\begin{abstract}
The progressive advancement of societies as a result of globalization establishes a competitive scenario in which the individual must seek continuous improvement and compliance with professionalization standards. Therefore, the objective of this research is to know the repercussions of the application of the new educational model in secondary education institutions on educational quality. The sample consisted of 50 secondary school teachers, aged between 23 and 45 years. An instrument was applied with 4 signalitic variables and 36 variables of the study phenomenon, considered with a scale of decimal ratio; which were processed in the statistical programs SPSS and Statistica. The main results show that the evaluation of teaching performance is significant to highlight the application of didactic procedures that ensure student learning. In addition, as long as human development and socio-emotional education are promoted, satisfactory results will be obtained in the application of the new educational model. Therefore, it is proposed to carry out continuous improvement forums where students, teachers, executives, parents and social agents that derive in the creation of public policies participate.
\end{abstract}

Educational Quality, Educational Model, Teaching

Citación: VILLARREAL-SOTO, Blanca Margarita, RAMOS-JAUBERT Roció Isabel, CEPEDA-GONZALEZ, María Cristina, ESPERICUETA-MEDINA, Marta Nieves y ALMANZA-SALAZAR, Javier Alejandro. Aprendizajes clave y su impacto en el ámbito socioemocional del estudiante de nivel secundaria. Revista de Pedagogía Crítica. 2019, 3-10: 1-8

\footnotetext{
* Correspondencia al Autor (Correo electrónico: mnieves@ uadec.edu.mx)

$\dagger$ Investigador contribuyendo como primer autor.
} 


\section{Introducción}

La importancia de la investigación educativa contribuye a la creación de propuestas de mejora del sistema educativo, proponiendo cambios significativos dentro de la curricula y el perfil de los diversos actores del fenómeno educativo. $\mathrm{Su}$ trascendencia dentro de diversos campos también ha impactado a los diversos investigadores de las ciencias sociales por medio de estudios metodológicos y estadísticos, los cuales ofrecen una perspectiva cuantitativa de los resultados educativos obtenidos a través de los años en diversos escenarios geográficos.

La presente investigación contribuye a este paradigma realizando un análisis de la calidad educativa dentro del nuevo modelo educativo mexicano para la educación obligatoria, el cual comenzará a implementarse a partir del ciclo escolar 2018-2019 en todos los centros de educación básica y media básica del país. Este modelo, el cual tiene como centro a la escuela, cuenta con el sustento de diversas corrientes humanísticas y cognitivistas, presenta un nuevo esquema de trabajo para el personal docente y administrativo de cada plantel, proponiendo retos $\mathrm{y}$ metas que deben ser cumplidos durante un corto tiempo para que su implementación sea exitosa.

Esta investigación se encarga de reunir los datos estadísticos de una población de docentes y sus conocimientos y perspectivas de dicho fenómeno, entrelazando las variables calidad de vida y calidad educativa, las cuales se presentan como contraste de esta investigación.

Junto a la ponderación de datos, se realizó un estudio comparativo y descriptivo de las variables con el fin de buscar validez y confianza de esta investigación.

Acompañado del sustento teórico, se presenta a la vez una serie de discusiones que contrastan los postulados con los resultados obtenidos, estableciendo generalidades que pueden ser extrapoladas a poblaciones con características similares a la observada. Dichas conclusiones permitieron llegar a propuestas de acción e intervención que pueden ser aplicadas en cualquier escenario educativo donde se busque llevar a la práctica los establecido en esta investigación

\section{Planteamiento de la Investigación}

\section{Objetivo General}

Conocer las repercusiones que tiene en la calidad educativa la aplicación del nuevo modelo educativo en las instituciones de educación secundaria de la ciudad de Saltillo, Coahuila.

\section{Objetivos Específicos}

- Analizar la aplicación metodológica de los nuevos planes y programas de estudio

- Establecer las funciones de la figura docente dentro del nuevo modelo educativo

- Inquirir las relaciones existentes entre la calidad de vida y la calidad educativa

\section{Objetivos Estadísticos}

- Frecuenciar el comportamiento que tiene el género dentro de la población

- Caracterizar la calidad educativa y su relación con la aplicación del nuevo modelo educativo

- Integrar las estructuras subyacentes del fenómeno y la calidad de vida

\section{Justificación}

La presente investigación ratifica su importancia en consideración que el fenómeno de la implementación del nuevo modelo educativo y sus vertientes en planes y programas es un innovador de la calidad educativa.

Los principales beneficiarios son los docentes de educación secundaria de planteles públicos y privados del centro de Saltillo en consideración que a través de los resultados de la investigación se podrán realizar diversas propuestas didácticas aplicables dentro de las nuevas estrategias sugeridas. Sin embargo, todos los docentes de instituciones de educación secundaria de Saltillo serán beneficiarios a través de las estrategias de intervención que resultarán de esta investigación.

A la vez se espera que todos los docentes de educación secundaria sean beneficiados al darse a conocer las generalidades y resultados de esta investigación. 
El valor teórico de esta investigación radica en la integración bibliográfica consultada para realizar un estudio integral sobre el nuevo modelo educativo y sus aplicaciones.

El valor metodológico radica en la elaboración de un instrumento que muestre la dinámica entre la muestra y el contraste del tema de la investigación.

\section{Sustento teórico}

El avance progresivo de las sociedades como resultado de la globalización, establece un escenario de competencia en el que el individuo debe buscar su mejora continua y el cumplimiento de estándares de profesionalización. Este proceso, obliga a los diversos medios e instituciones que preparan a los individuos a encontrarse en constante actualización. En el caso de la educación, los diversos sistemas educativos declinan por contenidos transversales y competencias disciplinarias que contribuyen a la construcción de una ideología de alcance mundial por medio de una educación de calidad.

Por lo anterior estudios como el de Horswood, D., Baker, J., Fazel, M., Rees, S., Heslop, L., \& Silove, D. (2019) sobre Factores escolares relacionados con lo emocional resultados de bienestar y reasentamiento de estudiantes de entornos de refugiados: protocolo para una revisión sistemática en donde se tiene evidencia de que al comprender qué elementos del clima escolar son influyentes en la salud mental y el reasentamiento de los estudiantes, los departamentos de educación y las escuelas pueden canalizar efectivamente su tiempo y recursos limitados para apoyar adecuadamente la población vulnerable.

La calidad educativa de la educación primaria ha sido analizada por diferentes autores, Acuña Gamboa, L. A. (2016) sostiene que el uso reiterado de este concepto en documentos emitidos por los Organismos Internacionales asume diversos significados, los cuales se reproducen de manera acrítica en los planes de desarrollo nacional y sectorial, colocando en un segundo plano la lectura de la realidad social. Por lo que concluye que este proceder responde a los lineamientos de corte internacional pero poco aporta al cambio y la mejora educativa en el país.
Siguiendo con el tema de calidad educativa en un estudio que busca entender la relación estudiante-institución educativa internacional realizado por Bordia, S., Bordia, P., Milkovitz, M., Shen, Y., \& Restubog, S. L. D. (2019) se examinó el contenido, la formación y el cumplimiento de su contrato psicológico. En donde los resultados arrojados indican que los estudiantes internacionales utilizan fuentes sociales e institucionales para crear el contrato psicológico, el cual, cuando se cumplió, conduce a resultados educativos y psicológicos positivos, en otras palabras, el que los docentes y el propio estudiante tenga conocimiento del contrato psicológico ayudará a las escuelas a gestionar mejor la relación estudiante-institución y por ede la calidad educativa.

Reynolds, A. J., Ou, S. R., Mondi, C. F., $\&$ Hayakawa, M. (2019) realizaron un estudio en el que se revisa lo relacionados con la escuela y los procesos familiares que conducen a efectos de intervención a largo plazo en el que se establece que la ganancias de aprendizaje influye en los programa, la escuela y la familia ya que parten de las políticas estatales para apoyar a los niños de preescolares que tienen necesidades especiales en las aulas de inclusión de preescolares en el estado

Rose, D., Jones Bartoli, A., \& Heaton, P. (2019) en su estudio para medir el impacto del aprendizaje musical en el desarrollo del bienestar cognitivo, conductual y socioemocional en los niños los resultados mostraron una asociación significativa entre la aptitud musical y la inteligencia en general no encontrando efectos para la memoria, ni para la integración visual motora o el comportamiento socioemocional, en donde por ejemplo el uso novedoso de la batería de evaluación de movimiento para niños proporciona evidencia de que el aprendizaje musical puede apoyar el desarrollo de la capacidad de un niño para juzgar la distancia, considerar la velocidad, concentrarse y usar sus sistemas nervioso propioceptivo, interoceptivo y exteroceptivo. A lo largo de la historia de la educación en México, esta búsqueda por una educación de calidad se ha visto reflejada en el establecimiento de nuevas reformas y modelos educativos que tienen el fin de adecuar los diversos procesos y legislaciones, así como establecer relaciones proactivas entre los diversos agentes que participan en el proceso educativo, tales como docentes, padres de familia, estudiantes y el entorno.

VILLARREAL-SOTO, Blanca Margarita, RAMOS-JAUBERT Roció Isabel, CEPEDA-GONZALEZ, María Cristina, ESPERICUETAMEDINA, Marta Nieves y ALMANZA-SALAZAR, Javier Alejandro. Aprendizajes clave y su impacto en el ámbito socioemocional del estudiante de nivel secundaria. Revista de Pedagogía Crítica. 2019 
El gobierno mexicano, en el Plan Nacional de Desarrollo 2013-2018 (PND 20132018) se ha propuesto construir un México con educación de calidad para lo cual-... propone implementar políticas de Estado que garanticen el derecho a la educación ... fortalezcan la articulación entre niveles educativos, y los vinculen con el quehacer científico, el desarrollo tecnológico y el sector productivo ...(Gobierno de la República, 2013, p. 59). La educación básica debe ser un punto focal en la formación del individuo, pues es la que brinda herramientas, habilidades y disciplina para poder desarrollarse durante las próximas etapas de su educación. La educación secundaria es la tercera etapa dentro de la educación básica y obligatoria, la cual da continuidad a la educación primaria y es sucedida por el bachillerato. Es por eso que la educación secundaria pretende lograr que los alumnos egresados del nivel de secundaria consoliden un conjunto de rasgos y competencias, no sólo cognitivos, y de manejo de la información, sino también aprendizajes y experiencias relacionados con lo afectivo, la convivencia y el desenvolvimiento de la vida en sociedad; que les permitan desenvolverse con éxito en un mundo de constante cambio.

\section{Metodología}

\section{Interrogante de investigación}

¿Cómo repercute en la calidad educativa la aplicación del nuevo modelo educativo en las instituciones de educación secundaria de la ciudad de Saltillo, Coahuila?

\section{Preguntas de investigación}

- ¿Cuál es la relación de la aplicación metodológica de los nuevos planes y programas de estudio con el ámbito socioemocional?

- ¿Cuáles son las funciones de la figura docente dentro del nuevo modelo educativo?

- $\quad$ ¿Cuál es la relación existente entre la calidad de vida y la calidad educativa?

\section{Preguntas estadísticas}

- $\quad$ ¿Cuál es el comportamiento que tiene el género dentro de la población?

- ¿Cuál es la relación de la calidad educativa respecto a la aplicación del nuevo modelo educativo?
¿Cómo se integran las estructuras subyacentes del fenómeno y la calidad de vida

\section{Muestra}

50 docentes de planteles de educación secundaria de carácter público y privado del centro de Saltillo, Coahuila

\section{Características de los sujetos}

Los sujetos que participarán en la aplicación del instrumento creado para esta investigación son docentes de escuelas secundarias del centro de la ciudad de Saltillo, Coahuila; el género de los sujetos son en mayoría mujeres oscilando entre la edad de 23 a 45 años. Dichos participantes muestran conductas un tanto diversas, desde la imposición de un respeto severo hasta un sentido de la confianza con sus alumnos. La mayoría de ellos se muestra preparados al momento de estar frente al grupo, indicando la posesión de un dominio del tema y planificación anticipada.

\section{Conceptualización de ejes}

Modelo Educativo: Orientación pedagógica que rige a un sistema educativo dentro de la elaboración de programas de estudio y sistematización de procesos de enseñanza y aprendizaje.

Calidad Educativa: Efectos positivamente valorados por la sociedad respecto del proceso de formación que llevan a cabo las personas dentro de su formación académica.

Calidad de Vida: Percepción que un individuo tiene dentro del contexto económico, social, cultural en los que vive y en relación con sus objetivos y sus normas

\section{Instrumento}

El instrumento que se utilizó posee del lado izquierdo en la parte superior el logo de la universidad, del lado derecho el escudo de la faculta de Ciencias, Educación y Humanidades, así como el nombre de estas dos instituciones, posteriormente se encuentran las variables signalíticas edad, género, ultimo grado de estudios y tipo de institución en que se labora. 
Debajo de éstas se pueden encontrar las instrucciones para contestar la encuesta. La escala, que representa cada uno de los números va desde el 0 hasta el 10 , donde el 0 indica la ausencia de frecuencia y el 10 la máxima frecuencia. Al final, se agradece a todas las personas que ayudaron a que esta investigación sea posible.

\section{Prueba piloto}

Una vez que se construyó el primer instrumento, fue aplicado a una muestra de 20 sujetos. Tras esta aplicación, se mostró que el resultado de las observaciones arrojaba resultados altos a la prueba de ítems, sesgando la información. Se revisó nuevamente los ítems reconstruyendo la operacionalización de variables, pasando a una nueva aplicación de 20 observaciones, donde se pudo notar resultados favorables para validar el instrumento.

\section{Procesamiento de información}

Con el objetivo de dar explicación a los ejes de investigación que conforman el fenómeno de estudio, se procesaron las frecuencias del instrumento de investigación a través del programa Statistic con la finalidad de caracterizar tanto a la población como al fenómeno de estudio a través de: frecuencias y porcentajes, caracterización y correlación.

Con el objeto de trabajar con exactitud y precisión, se desarrolló un análisis entre medias y medianas encontrando que no había diferencias significativas, por lo tanto, se procedió a trabajar con estadística paramétrica.

\section{Resultados}

\section{Frecuencias y Porcentajes}

A continuación, se presenta en análisis de las variables signalíticas género, edad, ultimo grado de estudios y tipo de institución en que labora.

\section{Variable Género}

En la tabla inferior se presenta el procesamiento de información de la variable género, la cual se encuentra representada por las categorías masculino y femenino.
Se observa que la población la constituyen 50 docentes de escuelas secundarias, en los cuales se indica respecto a la variable género que 26 son del género masculino, representando a un $52 \%$ de la población, mientras que el $48 \%$ está representado por el género femenino. Se infiere que la extrapolación con el mayor grado de validez es para el género masculino, pues es el que se encuentra con mayor representación T de Student

Con la finalidad de comparar muestras a través de sus medias aritméticas y encontrar diferencias significativas dependiendo de la edad, genero, institución y semestre, se muestran a continuación análisis comparativos con la prueba t de student para muestras independientes con un valor probable de error menor a 0.05 $(\mathrm{p}<0.05)$, los valores estadígrafos que aparecen en este análisis son los valores de la muestra de cada grupo de análisis (Media (X) de los grupos), el valor de la prueba $\mathrm{t}$ (t-valor), los grados de libertad (df) y la probabilidad del nivel de error (p) . Para el primer comparativo se toma como variable agrupadora edad con estrategia didáctica.

Del análisis se observa que las variables, juego de roles y uso de computadora destacan en el comparativo donde se observa que los alumnos de 15 años trabajan mejor por medio de la estrategia didáctica de juego de roles, por otro lado, podemos ver que los estudiantes de esta edad se familiarizan más con el uso de computadoras para su actividad escolar. Por lo tanto, se infiere que los alumnos de bachillerato dominan mejor los recursos tecnológicos que se le asignan a su institución, siendo así una gran ayuda para poder tener una mejor actividad escolar. Como segundo comparativo se toma como variable agrupadora género con estilo de aprendizaje y estrategias didácticas

Del análisis se observa que dentro de los estudiantes de género masculino tienen un mayor uso de plataformas virtuales más adecuado el uso de la realización de líneas del tiempo como estrategia didáctica en comparación con las estudiantes de género femenino, pero por otro lado se observa que las alumnas buscan diferentes variantes de aprendizaje, ya que piensan que buscan otras alternativas en lugar de seguir la misma línea de trabajo tal y como se observa que los alumnos si la siguen como estilo de aprendizaje. 
Por lo tanto, se infiere que los estudiantes de sexo masculino realizan con mayor facilidad las actividades por medio del uso de plataformas virtuales, así como las estudiantes de género femenino realizan las actividades yéndose por la misma línea de trabajo, mientras que los de género masculino buscan alternativas para llegar a cumplir sus objetivos

Como tercer comparativo se toma como variable agrupadora institución con estilos de aprendizaje y estrategias didácticas.

Del análisis se observa que dentro de los dos bachilleratos los alumnos del Ateneo Fuente piensan que las mejores estrategias didácticas que pueden aplicar los docentes es por medio de proyectos, consultas, líneas del tiempo, apuntes, ensayos, así como creen que es más viable el uso de computadoras, la realización de discusiones, el aplicar tutorías y el aprendizaje por medio de simulaciones, mientras que los alumnos de la Mariano Narváez Turno Matutino dudan que esto sea de mayor impacto para su aplicación dentro del aula, por otro lado se puede apreciar que los alumnos del Ateneo Fuente buscan las razones detrás de cada hecho con el cual trabajan, así como examinan la información antes de ser procesada, a diferencia de los alumnos de la Mariano Narváez, los del Ateneo suponen que la vida es una aventura. Mientras que los alumnos de la Mariano buscan alternativas de aprendizaje así dejando ver que los alumnos del Ateneo buscan seguir la misma línea de trabajo, pero eso si realizan las actividades paso a paso.

Así que, se infiere que los estudiantes del Ateneo Fuente utilizan más estrategias didácticas $\mathrm{y}$, por lo tanto, identifican las actividades que realizan en el aula, así como realizan más reuniones de tutorías.

Como cuarto comparativo se toma como variable agrupadora semestre con estrategia didáctica y creatividad.

Del análisis se puede observar que los alumnos de segundo semestre optan por tomar la estrategia didáctica de juego de roles, así como también ven a los maestros como guía, por otro lado, los alumnos de cuarto semestre realizan sus actividades apoyándose de mejor manera con el uso de la imaginación.
Por lo tanto, se infiere que los alumnos de cuarto semestre tienen imaginación ya que, involucran situaciones por las que se han enfrentado, así siendo más agiles al momento de ser creativos, por otro lado, los alumnos de segundo semestre requieren más apoyo del docente como guía.

\section{Análisis Factorial}

Este análisis se realizó con el procedimiento de principales componentes que sustentan la propuesta de innovación, con rotación de factores varimax normalizado, con un nivel probable de error $\mathrm{p} \leq .05$, un $\mathrm{r}=0.17$ y un nivel de confianza de $99.95 \%$. Se obtienen 21 factores, con un eigenvalor mínimo de 1 , esta marca un total de $72.46 \%$ de explicación del fenómeno de estudio, el cual se exhibe el factor 2, siendo aquí donde se prospecta la propuesta de innovación educativa y los factores 4,6,8,9 y 13 lo sustentan.

La invención en la creatividad, su estilo de aprendizaje y su estrategia didáctica

El factor 2, denominado El uso integrado de los estilos de aprendizaje con las estrategias didácticas, potencia la creatividad desde la invención, la diversidad y esto hace que se fortalezca su imaginación, por otro lado, se puede inferir que los estudiantes de estos bachilleratos suponen que las estrategias didácticas de proyectos son importantes para la realización de clases, esto puede hacer que dentro del aula se implementen las actividades lúdicas, estas harán que se fortalezca la convivencia de los alumnos, por lo tanto, las exposiciones, actividades por competencias y diversas actividades áulicas serán de mayor impacto.

Por otro lado, argumentan que la realización de tareas, consultas, ensayos, resúmenes benefician el reforzamiento de las habilidades extracurriculares, así como la realización de actividades que les muestren diversas variantes que pueden ser implementadas para que sean de gran utilidad a menudo, así también sea información que les muestren las razones por las que están estudiando. $\mathrm{Y}$ es aquí donde se presenta la imaginación que los alumnos argumentan, ya que, es aquí donde esta se potencializa, así como la habilidad de invención por parte de los estudiantes. 


\section{Conclusiones}

El desarrollo de las estrategias pedagógicas tiene impacto en el contexto del desarrollo de los estudiantes en el aula, es por eso que es necesario que los docentes a la hora de desarrollar una clase estén conscientes de los diferentes procesos que los estudiantes deben desarrollar en el aula.

Los recursos tecnológicos y las estrategias de los docentes deben de salir a flote, ya que cada alumno tiene diferente forma de aprender, de recabar información y de resolver diferente problemática, no obstante que la formación de los estudiantes está guiada por las habilidades de los docentes, que están encargados de realizar actividades con diferente método, para llevar a cabo un mejor aprendizaje de los estudiantes.

A través de distintas estrategias de aprendizaje, el estudiante podrá desarrollar diversos recursos que le ayudarán dentro de sus procesos educativos $\mathrm{y}$, en general, a desarrollarse en cualquier contexto social.

Es importante que en los procesos educativos los estudiantes no solo desarrollen y adquieran nuevos conocimientos, sino la habilidad de generar sus propias ideas y su inteligencia emocional, a través de un acompañamiento socioemocional escolar reflejado en el desempeño satisfactorio del alumno y su desenvolvimiento en el entorno

\section{Referencias}

Acuña Gamboa, L. A. (2016). La calidad de la educación básica vista desde los Organismos Internacionales: El caso del BID, la OCDE y UNESCO. En L. A. Acuña Gamboa \& F. Santillán Campos(Coords.), La investigación científica en Chiapas: Enfoques y perspectivas regionales(pp.23-39). Guadalajara: CONACyT, Universidad Complutense de Madrid y CENID

Beckman. (2013). Modelos de estilos de aprendizaje: una. Bogotá, Colombia. : Revista Colombiana.

Bordia, S., Bordia, P., Milkovitz, M., Shen, Y., \& Restubog, S. L. D. (2019). What do international students really want? An exploration of the content of international students' psychological contract in business education. Studies in Higher Education, 44(8), 1488-1502.

Garcia. (2005). Estilos de aprendizaje. Estilos de aprendizaje, 2.

Goñi. (2005). una vercion critica del concepto de creatividad ACTUALIDADES INVESTIGATIVAS EN EDUCACION, 4.

Gobierno de la República. (2013). Plan Nacional de Desarrollo 2013-2018. Recuperado de http://pnd.gob.mx/wpcontent/uploads/2013/05/PND.pdf

Hernádez. (20 de noviembre de 2005). Rrevicion de literatura: Estilos de aprendizaje. Recuperado el 4 de Mayo de 2019, de https://www.researchgate.net/publication/32733 6234_Revision_de_literatura_estilos_de_apren dizaje

Horswood, D., Baker, J., Fazel, M., Rees, S., Heslop, L., \& Silove, D. (2019). School factors related to the emotional wellbeing and resettlement outcomes of students from refugee backgrounds: protocol for a systematic review. Systematic reviews, 8(1), 107

Hoz, A. c. (20 de Noviembre de 2005). Revición de literatura: Estilos de aprendizaje. Recuperado el 4 de mayo de 2019, de https://www.researchgate.net/publication/32733 6234_Revision_de_literatura_estilos_de_apren dizaje

Hoz, Z. c. (2015). Estilos de aprendizaje . Revición de estilos de aprendizaje, 3 .

Márquez. (4 de Noviembre de 2005). Rrevisión de literatura: estilos de aprendizaje. Recuperado el 5 de Mayo de 2019, de https://www.researchgate.net/publication/32733 6234_Revision_de_literatura_estilos_de_apren dizaje

Monrreal. (20 de Junio de 2005). UNA REVISIÓN CRÍTICA DEL CONCEPTO DE CREATIVIDAD. Recuperado el 5 de Mayo de 2019,

file:///F:/Extra\%20Innovaci\%C3\%B3n\%20educ ativa/Vista\%20de\%20Una\%20revisi\%C3\%B3n $\% 20 \mathrm{cr} \% \mathrm{C} 3 \%$ ADtica\%20del\%20concepto\%20d e\%20creatividad.html. 
Reynolds, A. J., Ou, S. R., Mondi, C. F., \& Hayakawa, M. (2019). 10 School-Related and

Rose, D., Jones Bartoli, A., \& Heaton, P. (2019). Measuring the impact of musical learning on cognitive, behavioural and socio-emotional wellbeing development in children. Psychology of Music, 47(2), 284-303.

Family Processes Leading to Long-Term Intervention Effects. Sustaining Early Childhood

Learning Gains: Program, School, and Family Influences, 235.

Schmeck, c. p. (1996). Los estilos de aprendizaje y el rendimiento en Ciencias Sociales. Anales de psicología, 156-166.

Torres. (20 de Junio de 2005). UNA REVISIÓN CRÍTICA DEL CONCEPTO DE CREATIVIDAD. Recuperado el 4 de Mayo de 2019,

de

file:///F:/Extra\%20Innovaci\%C3\%B3n\%20educ ativa/Vista\%20de\%20Una\%20revisi\%C3\%B3n $\% 20$ cr\%C3\%ADtica\%20de1\%20concepto\%20d e\%20creatividad.html

Trigo. (2005). UNA REVISIÓN CRÍTICA DEL CONCEPTO DE CREATIVIDAD. ACTUALIDADES INVESTIGATIVAS EN EDUCACION, 4
VILLARREAL-SOTO, Blanca Margarita, RAMOS-JAUBERT Roció Isabel, CEPEDA-GONZALEZ, María Cristina, ESPERICUETAMEDINA, Marta Nieves y ALMANZA-SALAZAR, Javier Alejandro. Aprendizajes clave y su impacto en el ámbito socioemocional del estudiante de nivel secundaria. Revista de Pedagogía Crítica. 2019 